\title{
Kératokyste odontogène périphérique
}

\author{
Tommaso Lombardi ${ }^{1}$, Marlène Schear ${ }^{2}$, Jacky Samson ${ }^{2, *}$ \\ Laboratoire d'Histopathologie buccale, École de Médecine dentaire, Genève, Suisse \\ Division de Stomatologie et Chirurgie orale, Ecole de Médecine dentaire, Genève, Suisse \\ jacky.samson@unige.ch
}

(Reçu le 4 janvier 2013, accepté le 18 février 2013)

Mots clés :

kératokyste odontogène

périphérique / kyste gingival

Key words:

peripheral odontogenic keratocyst / gingival cyst

\begin{abstract}
Résumé - Le kératokyste odontogène périphérique constitue une entité rare dont on retrouve seulement une quinzaine de cas dans la littérature. Son étiopathogénie est encore controversée. Pour certains auteurs, il est difficile de le différencier du kyste gingival de l'adulte; pour d'autres, il constitue l'équivalent périphérique du kératokyste odontogène osseux. Les données physiopathologiques et histopathologiques tendent à confirmer cette dernière hypothèse.

La présentation d'un cas permettra de mieux faire connaître cette lésion et de préciser les éléments cliniques et radiologiques qui doivent faire évoquer le diagnostic.
\end{abstract}

Abstract - Peripheric odontognic keratocyst. Peripheral odontogenic keratocyst is a rare entity with about only fifteen cases reported in the literature. Its pathogenesis is still controversial. For some authors, it is difficult to differentiate the gingival cyst of the adult, for others it is the gingival equivalent of the odontogenic keratocyst arising within the jawbones. Pathophysiological and histopathological data tend to confirm this hypothesis.

In presenting this case we wish to raise awareness of this lesion and to discuss the clinical and radiological features that should suggest the diagnosis.

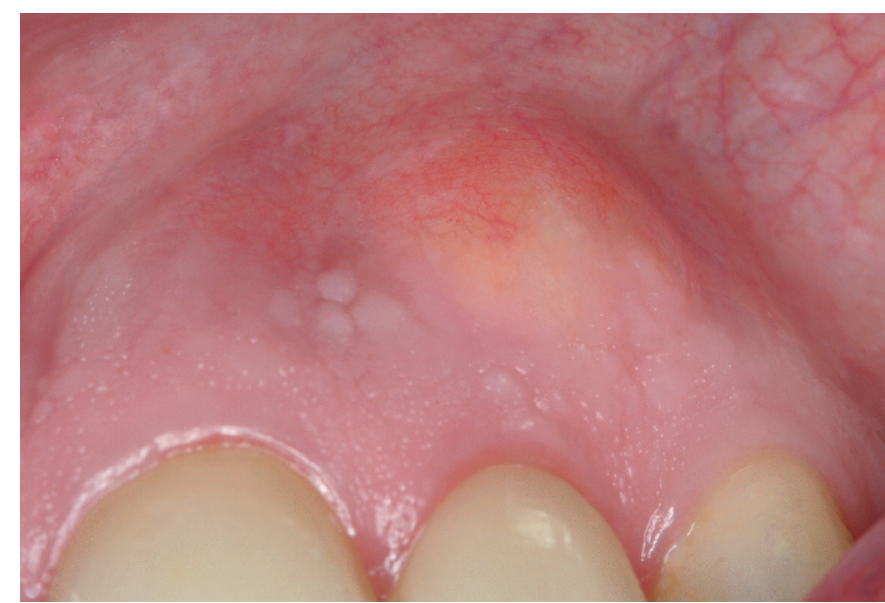

Fig. 1. Tuméfaction vestibulaire jaunâtre, fluctuante, indolore, située en regard de la racine de la 22 et de l'espace inter-radiculaire 22-23. Fig. 1. Vestibular fluctuating yellowish swelling, painless, opposite to the root of 22 and the inter-radicular space 22-23.

Une patiente, âgée de 72 ans, est venue consulter en octobre 2012 pour une lésion évoluant depuis 3 mois.
Il s'agissait d'une tuméfaction en verre de montre, jaunâtre, fluctuante, indolore, mesurant $10 \mathrm{~mm}$ dans le sens de la hauteur et $7 \mathrm{~mm}$ dans le sens de la largeur, siégeant à hauteur de la papille interdentaire 22-23, à $10 \mathrm{~mm}$ de son sommet (Fig. 1). Sur l'orthopantomogramme, il existe en regard de la lésion une discrète image radio-transparente, de forme ovalaire, à grand axe vertical sans limites marquées (Fig. 2). Lors de l'intervention, le décollement du lambeau muco-périosté a entrainé l'élimination d'un liquide crêmeux, homogène, jaunâtre. On a facilement identifié une fine paroi kystique qui ne présentait aucune réelle adhérence au périoste ou à l'os sousjacent. Après son élimination, on pouvait constater qu'elle n'avait aucun rapport avec les racines de 22 et 23 (qui étaient vivantes) et que le kyste avait entrainé une lyse de la corticale osseuse en forme de cupule. Un curetage appuyé de la surface osseuse a été réalisé comme on le fait systématiquement. 
L'examen histopathologique de la pièce d'exérèse (Fig. 3) a montré qu'il s'agissait d'une paroi kystique bordée par un épithélium à surface ondulée, par endroits parakératosique, dont l'assise basale palissadique avait une face profonde rectiligne. La paroi conjonctive fibreuse était par endroits le siège d'un dense infiltrat inflammatoire lymphoplasmocytaire en regard duquel l'épithélium perdait son aspect caractéristique. Dans la cavité, il persistait focalement, attaché à l'épithélium de bordure, un peu du contenu kystique représenté par des squames de kératine intriquées avec des polynucléaires. Cet aspect est caractéristique d'une paroi de kératokyste odontogène sans ilôts épithéliaux ou microkystes satellites. En raison du caractère superficiel de la lésion, il a été demandé à la patiente d'en assurer elle-même le contrôle à long terme.

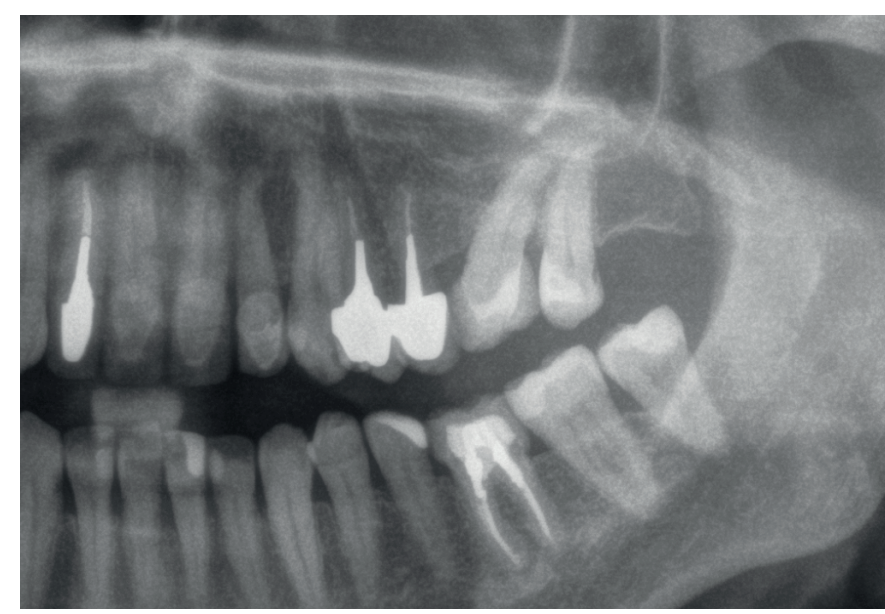

Fig. 2. Discréte image radiotransparente ovalaire, sans limites nettes, entre les racines de 22 et 23.

Fig. 2. Small radiolucent oval area, ill-limited, situated between the roots of 22 and 23.

Le kératokyste odontogène périphérique représente une entité rare dont on retrouve seulement une quinzaine de cas dans la littérature [4] ; dans les principaux traités de pathologie buccale, soit il est évoqué en deux lignes [2], soit il est ignoré [3]. Progressivement, son étiopathogénie a été précisée. Même si les 2 premiers cas ont été publiés en 1979 comme kyste gingival de l'adulte [1], il apparaît beaucoup plus proche du kératokyste osseux que du kyste gingival de l'adulte. C'est pourquoi on tend maintenant à le considérer comme l'équivalent périphérique du kératokyste odontogène car il en possède l'aspect histopathologique et les principaux caractères physiopathologiques (potentiel d'agressivité locale et de récidive) [2]. C'est une lésion du sujet âgé, le plus

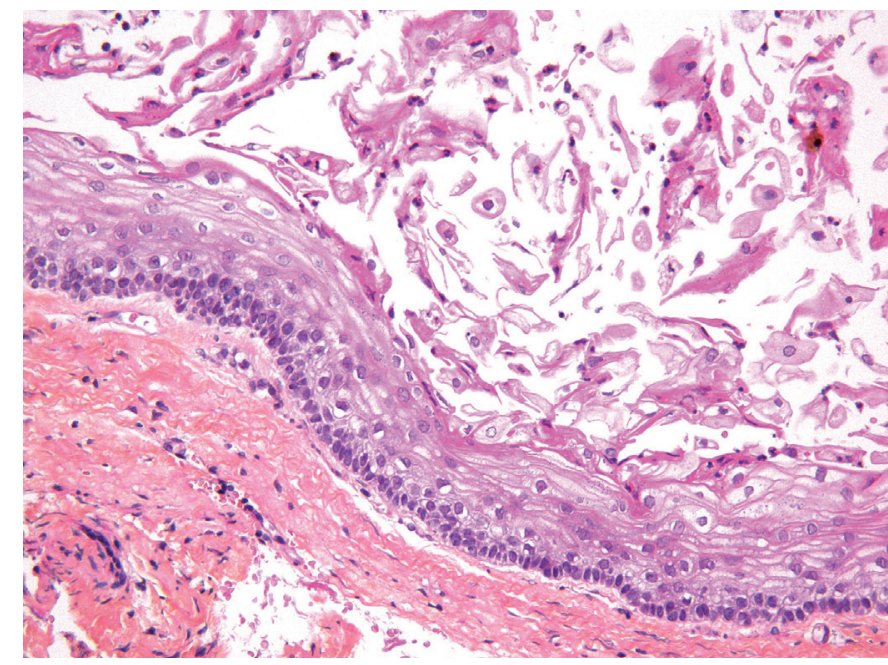

Fig. 3. Kératokyste parakératosique : l'assise basale est palissadique, à face profonde rectiligne. Squames de kératine dans la lumière du kyste (HES, ×20).

Fig. 3. Odontogenic keratocyst, parakeratinized type: well-defined basal layer of palisading columnar cells, absence of rete pegs. The lumen contains keratinized cells and debris (HES, $\times 20$ ).

souvent de sexe féminin comme le cas présenté. Cliniquement, le kyste gingival de l'adulte est normalement de plus petite taille et plus près du sommet de la papille inter-dentaire. Mais tous les deux, ils siègent surtout à la mandibule, dans la région antérieure. Tous les deux également, ils peuvent s'accompagner d'une lyse osseuse ; cette lyse osseuse reste toujours superficielle et elle n'est jamais comparable à celle observée avec le kyste parodontal latéral [2,3].

Le kératokyste odontogène périphérique possède des caractéristiques comparables à celles du kératokyste odontogène : il a le même aspect histopathologique et le même potentiel d'agressivité et de récidive, et il peut également être observé dans une naevomatose baso-cellulaire. Il semble donc bien constituer l'équivalent périphérique du kératokyste odontogène osseux [4].

\section{Conflits d'intérêt : aucun}

\section{Références}

1. Buchner A, Hansen LS. The histolomorphologic spectrum of the gingival cyst in the adult Oral Surg Oral Med Oral Pathol 1979;48:532-9.

2. Neville BW, Damm DD, Allen CM, Bouquot JE. Oral and maxillofacial pathology, 3rd ed. Saunders Elsevier, St Louis, 2009.

3. Regezi JA, Sciubba JJ, Jordan RCK. Oral pathology. Clinical pathologic correlations, 5th ed. Saunders Elsevier, St Louis, 2008.

4. Schaer M, Lombardi T. Kératokyste odontogéne périphérique : rappel embryologique et revue de la littérature. Med Buccale Chir Buccale 2013;19:101-8. 